\title{
Birthweight and fasting glucose and insulin levels: results from the Aboriginal Birth Cohort Study
}

\section{Susan M Sayers FAAP, FRACP, PhD Associate Professor, Child Health Division \\ Susan A Mott BSc, RN, MPH, Senior Research Officer ${ }^{2}$ \\ Kay D Mann MMathStat Research Assistant \\ Mark S Pearce \\ $\mathrm{BSc}, \mathrm{MSc}, \mathrm{PhD}$, Reader in Lifecourse Epidemiology \\ Gurmeet R Singh \\ PhD, MD, FRACP Senior Research Fellow \\ 1 Menzies School of Health Research, Darwin, NT. \\ 2 Centre for Chronic Disease, School of Medicine University of Queensland Brisbane, QLD. \\ $\mathbf{3}$ Institute of Health and Society, Newcastle University Newcastle upon Tyne, UK \\ sue.sayers@ \\ menzies.edu.au}

MJA 2013; 199: 112-116 doi: 10.5694/mjal3.10200

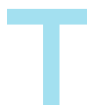

he concerning rise in type 2 diabetes in Indigenous populations continues, with different hypotheses put forward to explain the phenomenon. International evidence links the fetal nutrition proxies of low birthweight (LBW) and fetal growth restriction (FGR) to chronic diseases in adult life. ${ }^{1}$ The developmental origins of health and disease (DOHaD) hypothesis states that undernutrition in utero results in permanent changes through epigenetic mechanisms that later influence disease development (http://www.mrc. soton.ac.uk/dohad). The highest risk for type 2 diabetes is reported when LBW or FGR is followed by later overweight or obesity, suggesting a mismatch between intrauterine and postnatal nutrition. $^{2}$

Despite recent improvements, Australian Aboriginal LBW rates remain double those of the non-Aboriginal population. ${ }^{3}$ National Aboriginal rates of FGR are unknown, but in a Northern Territory study, 25\% of Aboriginal newborns were defined as fetal growth restricted. ${ }^{4}$

Concurrently, not only are Australian Indigenous rates of overweight and obesity increasing - they currently range from $37 \%$ for ages $15-24$ years to $74 \%$ for those aged over 55 years ${ }^{5}$ - but $10 \%-30 \%$ of Aboriginal people are now estimated to have type 2 diabetes. ${ }^{6}$

The high-risk combination of LBW and later obesity has been shown to be the greatest risk for elevated blood pressure in a cross-sectional community study of a NT Aboriginal population. ${ }^{7}$ More recently, a retrospective study in one NT community linked LBW and later chronic disease in Australian Aboriginal people ${ }^{8}$ and suggested LBW may be a contributor to the current high rates of type 2 diabetes in the Aboriginal population.

Using data from a prospective lifecourse Aboriginal birth cohort study, our aim was to examine the relationships of glucose and insulin metabo-

Objective: To examine the relationships between birthweight, current size, and fasting glucose and fasting insulin levels in Aboriginal adolescents.

Design, participants and setting: Longitudinal prospective study of a Northern Territory Aboriginal birth cohort of 686 Aboriginal babies born at the Royal Darwin Hospital between January 1987 and March 1990, and followed up between December 2006 and January 2008 in over 40 NT locations.

Main outcome measures: Fasting insulin and glucose levels, adjusted for gestational age, sex and contemporary age.

Results: Among the 134 participants with complete data, those with fetal growth restriction (FGR) or low birthweight (LBW) at birth were not overweight at 18 years. In these circumstances, birthweight showed a significant positively directed association with fasting glucose levels $(P=0.002)$. Current weight showed a significant and positively directed association with both fasting insulin $(P<0.001)$ and fasting glucose levels $(P=0.001)$, and current height showed a significant and positively directed association with insulin levels $(P=0.006)$.

Conclusions: Birthweight was only positively associated with fasting glucose levels, with no association with fasting insulin levels. The high-risk combination for type 2 diabetes of LBW or FGR with later overweight or obesity was rare in this adolescent Aboriginal population.

lism with birth and current adolescent size.

\section{Methods}

We conducted a prospective lifecourse study of an NT Aboriginal birth cohort.

The recruitment and follow-up of this birth cohort has been previously published. ${ }^{9,10}$ In brief, 686 Aboriginal babies out of a possible 1238 born at the Royal Darwin Hospital (19871990) were recruited into the study. There were no significant differences in the mean birthweight, sex ratio or birthweight frequencies between those recruited (686), those with gestational age estimation (603) and those not recruited. At follow-up (2005-2008) in over 40 NT locations, 68 participants could not be found $(9.9 \%)$. Of the remainder, 27 had died, 11 refused consent, 111 were traced but were unable to be examined for logistical reasons, ${ }^{10}$ and 469 who had complete perinatal and adolescent data were examined.

The birth measures of weight, gestational age estimations and follow-up anthropometric measures have previously been described. ${ }^{4,10}$ Within 4 days of birth, the same neonatal paediatrician performed a gestational age assessment using the Dubowitz scoring system. ${ }^{11}$ LBW was defined as birthweight $<2500 \mathrm{~g}$, FGR as birthweight $<10$ th percentile for gestational age, and large for gestational age as birthweight $>90$ th percentile for gestational age, using an Australian population reference standard contemporary with cohort recruitment. ${ }^{12}$

Adolescents were measured in light clothing while barefoot. Height was measured to the nearest millimetre using a portable stadiometer and weight was measured to the last complete $0.1 \mathrm{~kg}$ with a digital electronic scale (TBF-521, Tanita).

Participants were asked to fast from midnight before the examination. Blood samples were taken at the time of examination, collected in fluoride oxalate tubes after application of anaesthetic cream to the venepuncture site, separated after collection (for a minority of samples, this was up to a maximum of 2-3 hours) and transported in cold-boxes to Darwin.

Fasting glucose levels were measured enzymatically using a modular analyser (Roche Diagnostics), fasting insulin levels were measured by 
immunoassay (AxSYM, Abbott Laboratories), and glycated haemoglobin concentrations by high-pressure liquid chromatography (PDQ, Primus Diagnostics). Insulin resistance was estimated from fasting insulin and glucose concentrations using homoeostatic model assessment (HOMA-IR). ${ }^{13}$

Cross-sectional growth outcomes were described by $z$ scores for weight for age (WAZ) and height for age (HAZ) using the 2000 Centers for Disease Control and Prevention (CDC) sex-specific growth reference. ${ }^{14}$ Undernutrition was defined as 2 standard deviations (SDs) below zero and overweight was defined as 2 SDs above zero, according to World Health Organization criteria. ${ }^{15}$

Fasting was defined as an overnight fast of 8 hours or more, and 134 participants satisfied this criterion and had complete perinatal and follow-up data.

Residence at the time of follow-up was defined as remote (residence in defined remote Aboriginal communities) or other (including the twin cities of Darwin and Palmerston and the greater Darwin area).

\section{Statistical analysis}

The clinical characteristics were summarised as means (SD), and if not normally distributed, as geometric means (SD) and as category percentages. Characteristics were compared between sexes using the $t$ test (for normally distributed values) and the $\chi^{2}$ test (for categorical values). Nonnormal data were transformed to yield normal distribution before $t$ tests were performed.

Representativeness of the fasting sample, as described above, was tested using $t$, Wilcoxon and $\chi^{2}$ tests, depending on the distribution of the test variable.

For analyses, birthweight, and adolescent height, weight and body mass index (BMI) were continuous variables, while birthweight for gestational age was dichotomised at the 10th percentile for FGR and at the 90th percentile for large for gestational age.

The relationships of fasting insulin, glucose and HOMA-IR measures to the birth size and current adolescent size were each analysed in standard regression models using the approach recommended by Lucas

\section{Comparison of the fasting sample and the original cohort: Aboriginal Birth} Cohort 1987-2008

\begin{tabular}{|c|c|c|c|}
\hline \multirow[b]{2}{*}{ Characteristic } & \multicolumn{2}{|c|}{ Mean (SD)* } & \multirow[b]{2}{*}{$P^{\dagger}$} \\
\hline & $\begin{array}{l}\text { Original cohort with } \\
\text { gestational age data } \\
\qquad(n=603)\end{array}$ & $\begin{array}{l}\text { Fasting participants } \\
\text { with complete data } \\
\qquad(n=134)\end{array}$ & \\
\hline Birthweight, g & $3013(654)$ & 3027 (689) & 0.78 \\
\hline Low birthweight ${ }^{\ddagger}$ & $18.2 \%$ & $16.4 \%$ & $0.54^{5}$ \\
\hline Gestational age, weeks & $38.74(1.96)$ & 38.67 (2.11) & $0.69^{9}$ \\
\hline Fetal growth restriction** & $27.5 \%$ & $24.6 \%$ & $0.39^{\$}$ \\
\hline Large for gestational age ${ }^{\dagger \dagger}$ & $7.8 \%$ & $9.7 \%$ & $0.35^{\S}$ \\
\hline \multirow[t]{2}{*}{ Male } & $52.9 \%$ & $44.0 \%$ & $0.28^{5}$ \\
\hline & $\begin{array}{l}\text { Remaining original } \\
\text { cohort at follow-up } \\
\qquad(n=469)\end{array}$ & & \\
\hline Current age, years & $18.30(1.09)$ & $18.14(1.12)$ & 0.06 \\
\hline Weight for age, $z$ score & $-0.63(1.62)$ & $-0.47(1.63)$ & 0.20 \\
\hline Height for age, $z$ score & $-0.28(0.92)$ & $-0.21(0.97)$ & 026 \\
\hline Body mass index, $\mathrm{kg} / \mathrm{m}^{2}$ & $21.49(5.63)$ & $21.68(5.47)$ & 0.63 \\
\hline Glycated haemoglobin & $5.20 \%(0.40 \%)$ & $5.18 \%(0.42 \%)$ & 0.49 \\
\hline C-reactive protein, $\mathrm{mg} / \mathrm{L}$ & $5.02(7.48)$ & $4.99(7.78)$ & 0.97 \\
\hline
\end{tabular}

$\mathrm{SD}=$ standard deviation. $*$ Unless otherwise specified. $\dagger t$ test unless otherwise specified. $\neq<2500 \mathrm{~g}$. $\$ \chi^{2}$ test. q Wilcoxon test. $* *$ Birthweight $<10$ th percentile for gestational age. $\uparrow \dagger$ Birthweight $>90$ th percentile for gestational age.

and colleagues ${ }^{16}$ adjusted for gestational age, sex, and contemporary age using Stata, version 11 (StataCorp).

In order to maintain the assumptions of regression-dependent variables, fasting insulin, glucose and HOMA-IR values were transformed using the natural log transformation. Each outcome variable was tested in separate univariate models for each of the birth measures (eg, model 1), then adjustment for height (eg, model 1a) or weight (eg, model $1 \mathrm{~b}$ ) was added to the model, then an interaction term between the birth measures and adolescent weight or height was added. Lastly, the outcome variables were tested in separate univariate models for adolescent height (eg, model 3) and weight (eg, model 4). Models were separately analysed with current-residence regression coefficients and were then back-transformed using exponentiation, presenting ratios for ease of interpretation.

The percentage of total variance in the outcome measures, accounted for by early life size and later adolescent size, were estimated by the difference in the coefficients of determination $\left(R^{2}\right)$ between fully adjusted birth models with and without the measure of interest.

\section{Ethics}

The Human Research Ethics Committee of the Northern Territory Depart- ment of Health and Families and Menzies School of Health Research, including the Aboriginal Ethics Sub Committee, which has the power of veto, approved the study. Written consent was obtained in the form of an itemised consent with participants allowed to refuse individual procedures.

\section{Results}

One hundred and thirty-four participants had complete perinatal and follow-up data and fasting insulin and glucose measures. Box 1 shows the comparison of the fasting subset with the complete cohort and the subset of participants who did not have fasting values. This fasting subset was not significantly different from the nonfasting subset in the perinatal measures, the follow-up measures and the levels of non-fasting biomarkers (glycated haemoglobin and C-reactive protein) associated with type 2 diabetes. There were 59 males and 75 females with a mean age of 18.14 (SD, 1.1) years. The birth and adolescent characteristics of this fasting subset are shown in Box 2. As expected, females were significantly shorter $(P<0.01)$ and lighter $(P<0.01)$. Only one participant had a fasting glucose $>7 \mathrm{mmol} / \mathrm{L}$ and there were no participants with fasting glucose values of 6.1-6.9 $\mathrm{mmol} / \mathrm{L}$. 


\section{Birth and current adolescent characteristics of 134 fasting Aboriginal adolescents}

\begin{tabular}{|c|c|c|c|c|}
\hline \multirow[b]{2}{*}{ Characteristic } & \multicolumn{3}{|c|}{ Mean (SD)* } & \multirow[b]{2}{*}{$P^{\dagger}$} \\
\hline & Total $(n=134)$ & Male $(n=59)$ & Female $(n=75)$ & \\
\hline \multicolumn{5}{|l|}{ Perinatal } \\
\hline Birthweight, g & 3027 (689) & $3178(730)$ & $2908(635)$ & 0.02 \\
\hline Gestational age, weeks & $38.7(2.1)$ & $38.7(2.4)$ & $38.6(1.9)$ & $0.79^{\ddagger}$ \\
\hline Low birthweight ${ }^{\S}$ & $16.4 \%$ & $10.2 \%$ & $21.3 \%$ & 0.08 \\
\hline Fetal growth restriction ${ }^{9}$ & $24.6 \%$ & $20.3 \%$ & $28.0 \%$ & 0.31 \\
\hline Large for gestational age** & $9.7 \%$ & $11.9 \%$ & 8.0 & 0.56 \\
\hline \multicolumn{5}{|l|}{ Adolescent } \\
\hline Age, years & $18.14(1.12)$ & $18.11(1.01)$ & $18.17(1.20)$ & 0.77 \\
\hline Weight, kg & $61.49(20.06)$ & $70.37(23.74)$ & $54.5(13.0)$ & $<0.01^{\ddagger}$ \\
\hline Weight for age, $z$ score & $-0.47(1.62)$ & $-0.24(1.74)$ & $-0.65(1.52)$ & 0.15 \\
\hline Weight for age, $z$ score $<-2^{1}$ & $17.9 \%$ & $18.6 \%$ & $17.3 \%$ & 0.84 \\
\hline Height, $\mathrm{cm}$ & $167.33(9.26)$ & $174.41(7.61)$ & $161.76(6.12)$ & $<0.01$ \\
\hline Height for age, $z$ score & $-0.21(0.97)$ & $-0.21(1.02)$ & $-0.21(0.95)$ & 0.98 \\
\hline Height for age, $z$ score $-2^{14}$ & $3.0 \%$ & $5.1 \%$ & $1.3 \%$ & $0.32^{\dagger+}$ \\
\hline Body mass index, $\mathrm{kg} / \mathrm{m}^{2}$ & $21.68(5.47)$ & $22.83(6.35)$ & $20.78(4.50)$ & $0.04^{\ddagger}$ \\
\hline Weight for age, $z$ score $>2^{15}$ & $6.7 \%$ & $13.5 \%$ & $1.3 \%$ & 0.09 \\
\hline Fasting glucose, mmol/L & $4.64(0.59)$ & $4.78(0.46)$ & $4.53(0.65)$ & $0.01^{\ddagger}$ \\
\hline Fasting insulin, mU/L & $8.36^{\ddagger \ddagger}(2.40)$ & 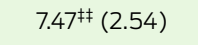 & $9.16^{\ddagger \ddagger}(2.28)$ & 0.19 \\
\hline HOMA-IR & $1.77^{\neq \ddagger}(2.53)$ & $1.58^{\ddagger \ddagger}(2.71)$ & $1.83^{\ddagger \ddagger}(2.39)$ & 0.38 \\
\hline Glycated haemoglobin & $5.16 \%$ \% $(1.08 \%)$ & $5.19 \%$ \% $(1.08 \%)$ & $5.14 \%$ \% $(1.08 \%)$ & 0.48 \\
\hline C-reactive protein, $\mathrm{mg} / \mathrm{L}$ & 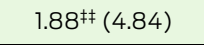 & $2.03^{\ddagger \ddagger}(4.07)$ & $1.78^{\ddagger \ddagger}(5.53)$ & 0.64 \\
\hline
\end{tabular}

HOMA-IR = insulin resistance estimated using homoeostatic model assessment. SD = standard deviation. * Unless otherwise specified. $\dagger t$ test unless otherwise specified. $\ddagger$ Unequal $t$ test. $\$<2500$ g. १ Birthweight $<10$ th percentile for gestational age.

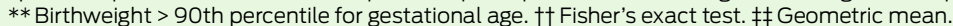

For the fasting dataset, using the international CDC reference, ${ }^{14}$ the mean WAZ scores were negative for both males and females. The proportions of males and females with WAZ $<-2$ (undernutrition) were $18.6 \%$ and $17.3 \%$, respectively. At follow-up, none of the 33 fasting adolescents who had been fetal growth restricted at birth had a WAZ > 2 (overweight/ obesity). Only one participant had a WAZ $>1$, with a similar profile occurring for those who had LBW.

Birthweight had a significant positively directed association with fasting glucose but not with fasting insulin levels. For every kg increment in birthweight, adolescent fasting glucose levels rose by $7 \%(P=0.002)$ (Box 3). This positively directed association of birthweight with fasting glucose levels remained after adjustment for adolescent height or weight, which accounted for $6 \%$ and $3 \%$ of the variation in fasting glucose levels, respectively.

The significant association with fasting glucose levels also remained when birthweight was categorised as birthweight for gestational age. For adolescents who had been fetal growth restricted at birth, fasting glucose levels were $7 \%$ less than for those who were not fetal growth restricted at birth $(P=0.003)$, and these relationships remained the same after adjustment for adolescent height and weight, again accounting for $6 \%$ and $3 \%$ of the variation in fasting glucose levels (Box 3).

Current height, weight and BMI had significant and positively directed associations with both fasting insulin and glucose levels in univariate models, with the regression ratio and percentage of variation explained being greatest for the fasting adolescent insulin and HOMA-IR measures.

For every $\mathrm{kg}$ increment in weight, $\mathrm{cm}$ increment in height or index point in BMI, fasting insulin levels rose by $3 \%, 3 \%$ and $9 \%$, respectively (Box 4 ), and HOMA-IR by 3\% (95\% CI, 1.021.04; $P<0.01), 3 \%$ (95\% CI, 1.01-1.06; $P=0.01)$ and $10 \%$ (95\% CI, 1.081.13), respectively (data for HOMAIR not shown), while for fasting glucose, the changes were $0.1 \%$ increase for every $\mathrm{kg}$ increment in weight, $0.2 \%$ for every $\mathrm{cm}$ increment in height, and $0.7 \%$ for every index point in BMI (Box 3).
Repeat analysis of all models with the inclusion of the remote status variable did not change any of the associations previously found. Regression models (models 3, 4 and 5 in Box 3 and Box 4) of height, weight and BMI were also reanalysed, with adjustment for birthweight, which did not change the results presented.

Apart from these main effects, there were positive and significant interactions between birthweight and height for insulin $(P=0.006)$ and HOMA-IR $(P=0.015)$ (data not shown). This allows for an extra increment in insulin and HOMA-IR values for participants who moved from higher birthweight to higher adolescent height. Hence, for a fixed current height, those adolescents with higher birthweights had higher measures of insulin and HOMA-IR values.

\section{Discussion}

In this adolescent cohort, there were no negatively directed associations between birthweight and either fasting glucose, insulin concentrations or the insulin-resistance measure, with no evidence of the U-shaped associations described for populations with similar high rates of type 2 diabetes in adult life. A positively directed association occurred for birthweight with fasting glucose levels. Consistent with these findings, those adolescents who had been fetal growth restricted at birth had lower fasting glucose concentrations. In contrast, even in this young and lean population, there were positively directed associations of current adolescent height and weight with fasting glucose and insulin concentrations and the insulin-resistance measure, albeit of a relatively trivial magnitude for fasting glucose. For fasting glucose levels, the effect of birthweight and current weight was similar $\left(R^{2}, 0.070 \mathrm{v} 0.076\right)$, but for fasting insulin levels, the effect of current weight was considerably stronger than birthweight $\left(R^{2}, 0.299 \mathrm{v} 0.019\right)$. These overall findings are similar to findings for these Aboriginal cohort participants at 11 years of age. ${ }^{17}$

The proportions of males and females with undernutrition (WAZ $<-2$ ) were $18.6 \%$ and $17.3 \%$, respectively, which far exceeded the expected $2.3 \%$ using the CDC sex-specific growth ref- 
erence. ${ }^{14}$ Hence, compared with this international reference, there was a marked excess of undernutrition in this cohort. Importantly, the findings of no associations between LBW and FGR with higher fasting adolescent insulin and glucose levels were contrary to the DOHaD hypothesis, and suggest that the adverse effects of poor fetal nutrition may be concealed by the persistent undernutrition in these Aboriginal adolescents.

The main strength of this study is that the data have been prospectively collected from a contemporary Australian Indigenous population. This is in contrast to many of the other studies examining the DOHaD hypothesis, which are retrospective studies.

Unusually for this type of cohort, at the time of recruitment less than $10 \%$ of mothers had homebirths. ${ }^{9}$ The singlepoint tertiary hospital recruitment meant birth measurements were standardised, and reliable gestational age estimations were all made by the one neonatal paediatrician within 4 days of birth. Hence, the better fetal growth surrogate of birthweight adjusted for gestational age was available for analysis. Further, all follow-up biological measures were directly collected.

The difficulty in obtaining reliable fasting blood samples in the field is reflected in the small sample size. However, a number of significant associations were present despite this sample size. A further limitation due to the age of participants is the necessity of using intermediary biomarkers of diabetes instead of the preferred specific disease end point. Socioeconomic status (SES) factors are potential confounders in this study and standard SES indicators were collected, such as years of schooling, house ownership and employment. There are limitations of standard measures in capturing the multidimensional differences within populations similar to this cohort population. ${ }^{18}$ In the absence of adequate discriminatory measures of SES, we used only remote current residence as an objective surrogate measure of SES. Including this variable made only a limited difference to the significance of one association.

Our findings are in contrast to the predominant literature of the $\mathrm{DOHaD}$

3 Size at birth and adolescent height and weight: relationships with fasting glucose $(\mathrm{mmol} / \mathrm{L})$ concentrations among Aboriginal adolescents $(n=134) *$

\begin{tabular}{llccc} 
Model & Ratio & $95 \% \mathrm{Cl}$ & $\boldsymbol{P}$ & $\boldsymbol{R}^{2 \dagger}$ \\
\hline Model 1: birthweight, kg & 1.07 & $1.03-1.11$ & 0.002 & 0.070 \\
Model la: adjusted for child height & 1.07 & $1.02-1.11$ & 0.005 & 0.057 \\
Model 1b: adjusted for child weight & 1.05 & $1.00-1.09$ & 0.035 & 0.030 \\
Model 2: FGR ${ }^{\ddagger}$ v non-FGR & 0.93 & $0.89-0.98$ & 0.003 & 0.062 \\
Model 2a: adjusted for child height & 0.93 & $0.89-0.98$ & 0.006 & 0.055 \\
Model 2b: adjusted for child weight & 0.95 & $0.90-0.99$ & 0.028 & 0.033 \\
Model 3: current height, cm & 1.002 & $0.99-1.01$ & 0.179 & 0.013 \\
Model 4: current weight, kg & 1.001 & $1.001-1.003$ & 0.001 & 0.076 \\
Model 5: body mass index & 1.007 & $1.003-1.01$ & $<0.001$ & 0.089 \\
\hline
\end{tabular}

FGR = fetal growth restriction. *All models adjusted for gestational age, sex and contemporary age. †Difference between fully adjusted birth models with and without the measure of interest.

$\ddagger$ Birthweight $<10$ th percentile for gestational age. $\oint$ Birthweight $\geqslant 10$ th percentile for gestational age.

\begin{tabular}{lcccc}
$\begin{array}{l}\text { 4 Size at birth and adolescent height and weight: relationships with fasting insulin } \\
\text { concentrations (mU/L) among Aboriginal adolescents }(n=134)^{*}\end{array}$ \\
Model & Ratio & $95 \% \mathrm{Cl}$ & $\boldsymbol{P}$ & $\boldsymbol{R}^{2 \dagger}$ \\
\hline Model 1: birthweight, $\mathrm{kg}$ & 1.28 & $0.94-1.73$ & 0.112 & 0.019 \\
Model la: adjusted for child height & 1.12 & $0.82-1.54$ & 0.471 & 0.004 \\
Model 1b: adjusted for child weight & 0.87 & $0.66-1.15$ & 0.326 & 0.005 \\
Model 2: FGR ${ }^{\ddagger}$ v non-FGR & 0.92 & $0.65-1.31$ & 0.656 & 0.002 \\
Model 2a: adjusted for child height & 1.01 & $0.71-1.43$ & 0.961 & 0 \\
Model 2b: adjusted for child weight & 1.27 & $0.94-1.71$ & 0.120 & 0.012 \\
Model 3: current height, cm & 1.03 & $1.01-1.05$ & 0.006 & 0.055 \\
Model 4: current weight, kg & 1.03 & $1.02-1.03$ & $<0.001$ & 0.299 \\
Model 5: body mass index & 1.09 & $1.07-1.12$ & $<0.001$ & 0.307 \\
\hline
\end{tabular}

FGR = fetal growth restriction. *All models adjusted for gestational age, sex and contemporary age. †Difference between fully adjusted birth models with and without the measure of interest.

$\neq$ Birthweight < 10th percentile for gestational age. $\$$ Birthweight $\geqslant 10$ th percentile for gestational age.

hypothesis describing inverse associations between birthweight and later fasting insulin and glucose levels. While these reports are mainly retrospective studies from developed populations, ${ }^{1}$ similar findings have been reported from Aboriginal populations ${ }^{19}$ and five low- or middle-income-country birth cohorts. ${ }^{20}$

Similar findings to our study have been reported in a young lean population of Guatemalan men and women at a mean age of 24 years. $^{21}$ Further, in the Newcastle Thousand Families Study, at 49-51 years of age, adult lifestyle factors explained larger proportions of variances for fasting and 2-hour glucose compared with early-life measures. ${ }^{22,23}$ A contemporary study of British children, based on maternal recall of birthweight, reports current size as the main determinant of insulin and glucose concentrations in childhood. ${ }^{24}$

The growth outcomes in our study suggest that a major nutritional mismatch between fetal and adolescent life has not occurred. It is likely that the permanent changes in response to undernutrition in utero have remained appropriate in this undernourished adolescent population with low rates of the high-risk combination of LBWor FGR-associated later obesity or overweight.

Given the high prevalence of overweight and obesity currently seen in the adult Aboriginal population, ${ }^{6}$ it is likely that the growth trajectory will positively change in this cohort and the high-risk combination for chronic disease of LBW or FGR followed by overweight or obesity will become more common over the next decade. Then the relationships of LBW or FGR with chronic biomarkers may become apparent, consistent with the DOHaD hypothesis. With the follow-up of this cohort at the age of 26 years currently underway, we are well placed to determine if and when the effects of poor intrauterine nutrition are potentiated by the onset of overweight and obesity. 
In the meantime, our findings suggest that the current high rates of type 2 diabetes observed in the adult Aboriginal population are more likely to be decreased by strategies targeted to improve lifestyle factors in childhood and adolescence, rather than those focusing on improving birthweight alone.

Acknowledgements: We thank the adolescents belonging to the cohort for their continued cooperation, and everyone who helped in the urban and rural locations. This work was supported by the National Health and Medical Research Council of Australia.

Competing interests: No relevant disclosures.

Received 15 Feb 2013, accepted 29 May 2013.

1 Barker DJP. Mother, babies, and health in later life. 2nd ed. Edinburgh: Churchill Livingstone. 1998.

2 Godfrey KM, Lillycorp KA, Burdge GC, et al. Epigenetic mechanisms and the mismatch concept of the developmental origins of health and disease. Pediatr Res 2007; 61 (5 Pt 2): 5R-10R.

3 Leeds K, Gourley M, Laws P, et al. Indigenous mothers and their babies, Australia 2001-2004. Canberra: AlHW, 2007. (AlHW Cat. No. PER 38; Perinatal Statistics Series No. 19.)

4 Sayers SM, Powers JR. Birth size of Australian Aboriginal babies. Med J Aust 1993; 159: 586-591.

5 Australian Bureau of Statistics, Australian Institute of Health and Welfare. The health and welfare of Australia's Aboriginal and Torres Strait Islander peoples. Canberra: Commonwealth of Australia, 2008. (ABS Cat. No. 4704.0; AlHW Cat. No. IHW 21.)
6 Fearnley E, Li SQ, Guthridge S. Trends in chronic disease mortality in the Northern Territory Aboriginal population, 1997-2004: using underlying and multiple causes of death. Aust NZ JPublic Health 2009; 33: 551-555.

7 Singh GR, Hoy WE. The association between birthweight and current blood pressure; a crosssectional study in an Australian Aboriginal community. Med J Aust 2003; 179: 532-535.

8 Hoy WE, Nicol JL. Birthweight and natural deaths in a remote Australian Aboriginal community. Med J Aust 2010; 192: 14-19.

9 Sayers SM, Mackerras D, Singh G, et al. An Australian Aboriginal birth cohort: a unique resource for a life course study of an Indigenous population. BMC Int Health Hum Rights 2003; 3: 1 .

10 Sayers S, Singh G, Mackerras D, et al. Australian Aboriginal Birth Cohort study: follow-up processes at 20 years. BMC Int Health Hum Rights 2009; 9: 23

11 Dubowitz LMS, Dubowitz V. A clinical manual: gestational age of the newborn. Philippines: Addison-Wesley, 1977.

12 Guaran RL, Wein P, Sheedy M, et al. Update of growth percentiles for infants born in an Australian population. Aust N Z J Obstet Gynaecol 1994; 34: 39-50.

13 Matthews DR, Hosker JP, Rudenski AS, et al. Homeostasis model assessment: insulin resistance and beta-cell function from fasting plasma glucose and insulin concentrations in man. Diabetologia 1985; 28: 412-419.

14 Kuczmarski RJ, Ogden CL, Grummer-Strawn LM, et al. CDC growth charts: United States. Advance Data 2000; 314: 1-27.

15 World Health Organization. Physical status: the use and interpretation of anthropometry. Report of a WHO Expert Committee. (WHO Technical Report Series No. 854.) Geneva: WHO, 1995.
16 Lucas A, Fewtrell MS, Cole TJ. Fetal origins of adult disease revisited. BMJ 1999; 319: 245-249.

17 Sayers S, Singh G, Mott S, et al. Relationships of birth weight and biomarkers of chronic disease in childhood: Aboriginal Birth Cohort Study 19872001. Paediatr Perinat Epidemiol 2009; 23 . 548-556

18 Braveman PA, Cubbin C, Egerter S, et al. Socioeconomic status in health research: one size does not fit all. JAMA 2005; 294: 2879-2888.

19 McNamara BJ, Gubhaju L, Chamberlain C, et al Early life influences on cardio-metabolic disease risk in aboriginal populations - what is the evidence? A systematic review of longitudinal and case-control studies. Int J Epidemiol 2012; 41: 1661-1682.

20 Norris SA, Osmond C, Gigante D, et al. Size at birth, weight gain in infancy and childhood, and adult diabetes risk in five low- or middle-income country birth cohorts. Diabetes Care 2012; 35 : 72-79.

21 Stein AD, Conlisk A, Torun B, et al. Cardiovascular disease risk factors are related to adult adiposity but not birth weight in young Guatemalan adults. J Nutr 2002; 132: 2208-2214.

22 Pearce MS, Unwin NC, Parker L, et al. Life course determinants of insulin secretion and sensitivity at age 50 years: the Newcastle Thousand Families Study. Diabetes Metab Res Rev 2006; 22: $118-125$.

23 Pearce MS, Unwin NC, Relton CL. Lifecourse determinants of fasting and post-challenge glucose at age 50 years: the Newcastle Thousand Families Study. Eur J Epidemiol 2005; 20: 915923

24 Whincup PH, Cook DG, Adshead F, et al. Childhood size is more strongly related than size at birth to glucose and insulin levels in 10-11-yearold children. Diabetologia 1997; 40: 319-326.

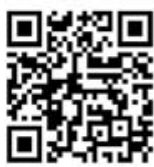

The MJA
No matter whether you are an Ansel Adams or just

a happy snapper, whether you use a high-end SLR

or a phone camera, the MJA invites subscribers to submit their digital images for our photography competition. Winning images will be published in the MJA Reflections section and on the MJA website.

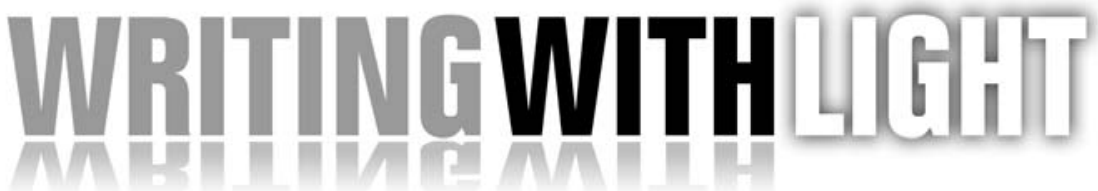

Images can be on any non-clinical subject matter and should be emailed to mjaphotos@mja.com.au in a high resolution .jpg format (approx $6 \times 4$ proportion). Images will not be judged on technical expertise alone, but also on subject matter, artistic merit and interest to readers.

Please include an image title (no more that 50 characters), along with your name and state of residence for publication. We will also require a phone contact number and email address for general correspondence.

\section{Competition}

email submissions to:

mjaphotos@mja.com.au

See page 136 for this issue's winning photo

For more details visit our website:

www.mja.com.au/author-centre/awards

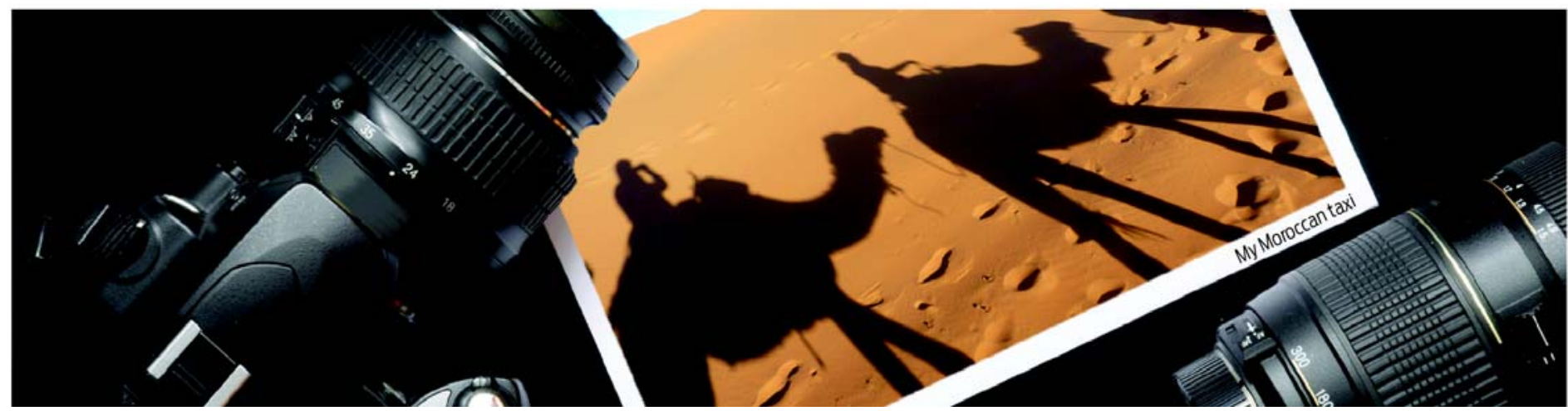

